\title{
TRANSFERT ARTISTICI NELL'ALTO ADRIATICO. NUOVE IPOTESI SUL SACELLO DI SAN PROSDOCIMO A PADOVA
}

\section{VALENTINA CANTONE}

\author{
UDC: $726.595 \cdot 033 \cdot 1(450.347)$ \\ $738.5033 .1 / .2$ \\ Original scientific paper \\ Manuscript received: 30. 11. 2015. \\ Revised manuscript accepted: 15. 03. 2016. \\ DOI: 10.1484/J.HAM.5.111328
}

V. Cantone Università degli Studi di Padova Dipartimento dei beni culturali: archeologia, storia dell'arte, del cinema e della musica, piazza Capitaniato, 7 35139 Padova, Italia

This paper focuses on the dome of the Early Christian chapel of saint Prosdocimus, in the church of Saint Justine, Padua. The radial iconography of the Ascension of Christ depicted during the middle of the $16^{\text {th }}$ century is considered a copy of the lost mosaic, realized in the early $6^{\text {th }}$ century, using as a model the dome of the neonian baptistery, in Ravenna, or other eastern similar layouts (Saint George and Saint Sophia in Thessaloniki). Today it is possible to reconsider this artistic transfer. In fact the modern frescoes are connected with the artistic renovatio of the martyrial space wanted by the abbot of the Benedictine Abbey of Saint Justine, during the years of the Council of Trent (1545-63). There is another reason which should be considered about the presence of the mosaic with the Apostles in the Early Christian dome. In fact a literary source of 1619 testifies that the Apostles were "insculpti", which means "done in relief". The archaeometrical analysis realized on a piece of white and fine mortar has demonstrated the presence of stucco in the chapel.

Keywords: Early Christian Art, Early Christian Mosaic, Saint Prosdocimus of Padua (Chapel), Iconography, Byzantine Mosaic, Restoration, Historiography.

\section{INTRODUZIONE}

"Le cupole, in quanto forme d'arte, se ne vanno in fretta", scrive John Shearman nel 1992, "e quella che noi dobbiamo ricostruire e interpretare è per lo più una vicenda storica frammentaria"'.

Il caso di studio che propongo tratta di una vicenda frammentaria in senso letterale. Infatti i mosaici che decoravano la cupola del sacello di San Prosdocimo a Padova sono contenuti all'interno di 115 cassette lignee, conservate nell'Abbazia di Santa Giustina.

A metà del Cinquecento il ciclo ornamentale venne abbattuto quasi integralmente a colpi di scalpello. Si tratta di un'eredità storica e artistica la cui preziosità è pari all'in- teresse scientifico che suscita e alla complessità di definire sul piano metodologico l'approccio o, meglio, gli approcci adeguati a trattare una materia così sconnessa e lacunosa ${ }^{2}$.

La datazione del mosaico è legata alla carica pubblica rivestita dal committente del sacello, che, secondo l'attuale orientamento della comunità scientifica, corrisponde al prefetto del pretorio Opilione, console nel $524^{3}$. L'ipotesi di datazione del sacello martiriale al primo ventennio del VI secolo coincide con il fiorire del culto per santa Giustina, iscritta nel canone ambrosiano proprio fra l'ultimo decennio del V secolo e il primo del secolo successivo ${ }^{4}$ e corrisponde alla realizzazione di un oratorio quasi gemello, per impostazione planimetrica e ornamentazione, dedicato a santa Maria mater Domini, affiancato alla basilica dei Ss. Felice e

${ }^{1}$ J. SHEARMAN, Only connect...Art and Spectator in the Italian Renaissance, Washington 1992, p. 150.

${ }^{2}$ M. TONZIG, La basilica e l'oratorio opilioniani e questioni relative, in EADEM, La basilica romano-gotica di santa Giustina in Padova, in "Bollettino del Museo Civico di Padova”, n.s., V (XXII)-VII, 1929 (1932), pp. 1-35; S. BETTINI, Padova e l'arte cristiana d'Oriente, in Atti del Reale Istituto Veneto di Scienze, Lettere ed Arti, Anno accademico 1936-1937, Tomo XCVI, Parte seconda, pp. 213-296; L. MICHELETTO, L'Oratorio paleocristiano di Opilione, in “Palladio. Rivista di storia dell'architettura”, n.s. anno IV, 1954, fascicolo IV (ottobre-dicembre), pp. 179-184; P.L. ZOVATTO, Mosaici paleocristiani delle Venezie, Udine 1963, pp. 44-49; IDEM, Decorazione dell'oratorio di S. Giustina, in P.L. Zovatto, N. Ivanoff, A. Sartori et al. (ed.), La basilica di Santa Giustina. Arte e storia, Castelfranco Veneto 1970, pp. 32-39; L. MICHELETTO, La basilica di Opilione: per un progetto di ricostruzione, in A. De Nicolò Salmazo e F.G. Trolese (dir.), I Benedettini a Padova e nel territorio padovano attraverso i secoli. Saggi storici sul movimento benedettino a Padova, pp. 299-306, in part. n. 175, pp. 304-305; M. SANNAZARO, Il complesso di Santa Giustina, in S. Lusuardi Siena, C. Forio Tedone et al. (ed.), Le tracce materiali del Cristianesimo dal tardo antico al Mille, in A. Castagnetti e G.- M. Varanini (dir.), Il Veneto nel medioevo. Dalla "Venetia" alla Marca Veronese, 2 voll., II, 1989, pp. 87-328, in part. pp. 232-244; G. Mackie, Early Christian Chapels in the West. Decoration, Function, and Patronage, Toronto 2003, pp. 41-44; E. CORTELLA, La basilica paleocristiana di Santa Giustina in Padova, Tesi di Specializzazione, Scuola di Specializzazione in Storia dell'arte e delle arti minori, Facoltà di Lettere e Filosofia, Relatore prof.ssa Giovanna Valenzano, A.A. 2005-2006, in part. pp. 43-55; Irina ANDREESCU TREADGOLD, Santa Giustina di Padova: considerazioni preliminari sui frammenti di mosaico parietale, in C. Angelelli (ed.), Atti del XIV colloquio dell'Associazione Italiana per lo Studio e la Conservazione del Mosaico, Tivoli 2009, pp. 349-358; Elisabetta CORTELLA, Modelli ravennati in territorio veneto: “Celeste palatium ac viridantia paradisi prata”, un'ipotesi iconografica per la decorazione parietale del sacello di san Prosdocimo in santa Giustina in Padova, in C. Caramanna, N. Macola e L. Nazzi (ed.), Citazioni, modelli, tipologie nella produzione dell'opera d'arte, atti della giornata di studio (Padova 29-30 maggio 2008), Padova 2011, pp. 19-27; V. CANTONE, A. SILVESTRI, G. MOLIN, Un approccio interdisciplinare per lo studio del mosaico medievale in area altoadriatica: i sacelli di san Prosdocimo a Padova e di santa Maria Mater Domini a Vicenza, in Atti del XX colloquio dell'Associazione Italiana per lo Studio e la Conservazione del Mosaico, in via di pubblicazione (2016).

3 The prosopography of the Later Roman Empire, ed. by J.R. Martindale, vol. II, AD 395-527, Cambridge 1980, pp. 807-809; G. PREVEDELLO, La Basilica di Opilione: cenni storici, in I Benedettini a Padova e nel territorio padovano attraverso i secoli, Padova 1980, Pp. 215-17; M. SANNAZARO, Il complesso di Santa Giustina, in A. Castagnetti e G.-M. Varanini (dir.), Il Veneto nel medioevo. Dalla “Venetia” alla Marca Veronese, Verona 1989, pp. 232-244; G. CUSCITO, Ancora sulle origini cristiane di Padova, in Un uomo chiamato Prosdocimo a Patavium, atti del convegno (Padova, Abbazia di Santa Giustina, 5 novembre 2011), a cura di F. Benucci (Antichità Altoadriatiche. LXXV), Trieste 2013, pp. 67-8o, in part. note 16 e 17.

${ }^{4}$ F. SAVIO, Gli antichi vescovi d'Italia, Milano 1916, pp. 930-935. 


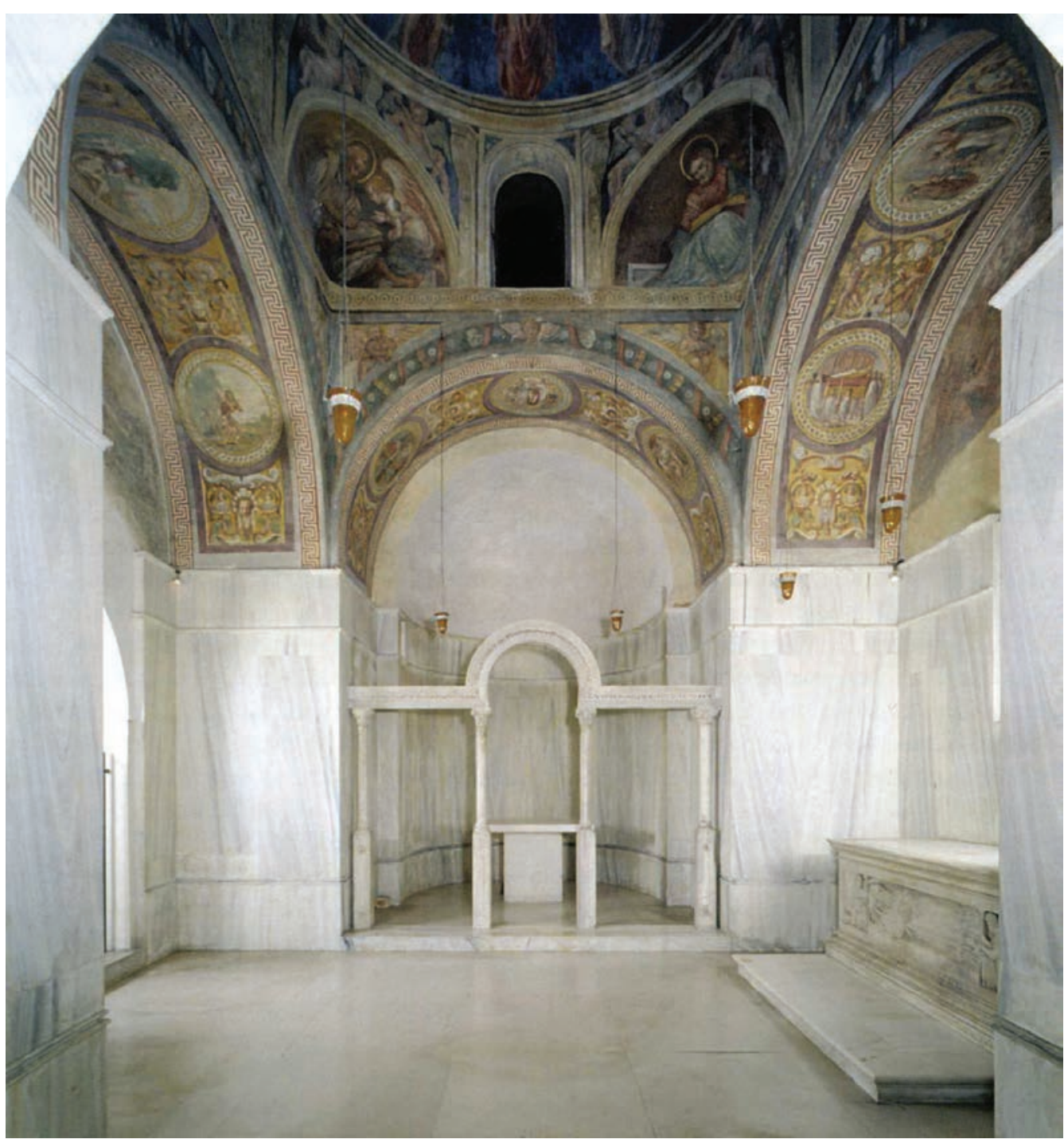

Fig. 1 Padova, Chiesa di Santa Giustina, sacello di San Prosdocimo, veduta absidale.

Fortunato di Vicenza, realizzata su committenza di un altro funzionario: il referendario Gregorio5.

Dal 2005, presso l'Università degli Studi di Padova, sono state condotte una serie di indagini archeometriche su duecento tessere vitree contenute nella cassetta numero 110. Essa contiene tremila tessere sciolte, raccolte a metà del Novecento sotto la pavimentazione del sacello di San Prosdocimo, insieme ad alcune centinaia di lacerti musivi.

Le analisi archeometriche condotte sui mosaici di Padova, i cui esiti sono stati raffrontati con i risultati delle indagini effettuate a Vicenza, hanno permesso di caratterizzare le complesse tecnologie di produzione e hanno evidenziato una buona comparabilità delle composizioni chimiche con i mosaici paleocristiani di Ravenna ${ }^{6}$.

I materiali padovani rivelano l'adozione delle più aggiornate tecnologie della produzione del vetro, suggerendo che Padova, città portuale legata all'Adriatico attraverso una fitta rete di canali navigabili, abbia importato gli stessi materiali e le medesime competenze comuni a Ravenna, che fra $\mathrm{V}$ e VI secolo è sede privilegiata di illustri cantieri musivi. A sua

\footnotetext{
${ }_{5}^{5}$ G. LORENZON, La basilica dei santi Felice e Fortunato di Vicenza, Vicenza 1938; A. PREVITALI, La decorazione del martyrion, in La Basilica dei santi Felice e Fortunato di Vicenza, 2 voll., Vicenza 1979, pp. 101-115; Il Veneto nel Medioevo; Ch. PIETRI, Aristocratie et société cléricale dans l'Italie chrétienne au temps d'Odoacre et de Théodoric, in Mélange de l'Ecole francaise de Rome Antique, I, Roma 1981, pp. 417-67, in part. p. 427; S. LUSUARDI SIENA, La costruzione del sacello di S. Maria Mater Domini (età gota), in A. Castagnetti e G.-M. Varanini (dir.), op. cit. (n. 3), pp. 196-204; G. MACKIE, op. cit. (n. 2), pp. 37-40. ${ }^{6}$ A. SILVESTRI, S. TONIETTO, G. MOLIN, The palaeo-Christian glass mosaic of St. Prosdocimus (Padova, Italy): archaeometric characterisation of "gold" tesserae, in Journal of Archaeological Science, 38, 2011, pp. 3402-3414; S. TONIETTO, F. NESTOLA, G.J. REDHAMMER, et al., Pyroxene inclusions in paleo-Christian mosaic tesserae: a tool for constraining the glass manufacturing temperature, in Applied Physics A, 103, 2011, pp. 207- 212; A. SILVESTRI, S. TONIETTO, G. MOLIN, et al., The palaeo-Christian glass mosaic of St. Prosdocimus (Padova, Italy): archaeometric characterisation of tesserae with antimony- or phosphorus-based opacifiers, in Journal of Archaeological Science, 39, 2012, pp. 2177- 2190; A. SILVESTRI, S. TONIETTO, F. D'ACAPITO, et al., The role of copper on colour of palaeo-Christian glass mosaic tesserae: an XAS study, in Journal of Cultural Heritage, 13, 2012, pp. 137-144; A. SILVESTRI, S. TONIETTO, G. MOLIN, et al., The palaeo-Christian glass mosaic of St. Prosdocimus (Padova, Italy): archaeometric characterisation of tesserae with copper- or tin-based opacifiers, in Journal of Archaeological Science, 2014, pp. 42, 51-67; A. ZOLEO, M. BRUSTOLON, A. BARBON, et al., Fe(III) and Mn(II) EPR quantitation in glass fragments from the palaeo-Christian mosaic of St. Prosdocimus (Padova, NE Italy): archaeometric and colour correlations, in Journal of Cultural Heritage, 16, 2015, pp. 322-328; A. SILVESTRI, S. TONIETTO, G. MOLIN, et al., The palaeo-Christian glass mosaic of St. Maria Mater Domini (Vicenza, Italy): multi-methodological study of tesserae and comparisons with coeval mosaic of St. Prosdocimus in Padova, in European Journal of Mineralogy, 27, 2015, pp. 225-245; A. SILVESTRI, F. NESTOLA, L. PERUZZO, Multi-methodological characterisation of calcium phosphate in late-antique glass mosaic tesserae, in Microchemical Journal, 124, 2016, pp. 811-818.
} 
volta, Padova, che nel medioevo era un «ineludibile crocevia d'accesso ai porti lagunari per tutto il Veneto centrale» ${ }^{7}$, ha costituito il punto di snodo di un transfert di materiali in transito verso Vicenza, probabilmente sfruttando la vocazione idrologica del territorio.

L'analisi comparativa delle tessere vitree di Padova e Vicenza ha inoltre dimostrato che per decorare il sacello di Santa Maria mater Domini sono state utilizzate in parte le stesse pizze di vetro usate nell'oratorio opilioniano, in parte vetri ricavati con tecnologie più conservative e materiali di riciclo.

Il rapporto tra il porto fluviale di Padova e quello marittimo di Ravenna non è solamente quello caratterizzato dalla circolazione di materiali e tecniche, sostenibile alla luce dai dati archeometrici. Esiste anche un altro legame, che pertiene più direttamente all'analisi storico artistica dei documenti materiali. Si tratta però di un legame molto più difficile da descrivere e circoscrivere, che si appoggia su una nuova valutazione critica delle fonti testuali e sui dati preliminari offerti da recenti indagini alle malte.

\section{SCAVI E RESTAURI NOVECENTESCHI}

Il sacello di San Prosdocimo, situato nella basilica di Santa Giustina, appare oggi dominato dal nitore delle lastre di rivestimento posate a metà del Novecento (fig. 1).

Il marmo di Lasa, situata tra Merano e la val Venosta, ha sostituito il ben più vibrante proconnesio, conservato solo in un paio di frammenti, tagliati e ricollocati. La chiarezza della planimetria, fatta da un quadrato di 4 metri scarsi di lato, disegnato dall'incrocio dei due bracci, la geometria lineare della pergula, l'isolamento acustico di cui gode l'ambiente, separato dalla basilica cinquecentesca dal corridoio dei martiri, contribuiscono a definire uno spazio in perfetto stile neopaleocristiano (fig. 2). Tanto che la comunità ortodossa ne chiede frequentemente l'uso per la liturgia battesimale, a conferma del fatto che il sacello martiriale viene percepito come autentico dai non specialisti.

In realtà, l'aspetto attuale del piccolo santuario costituisce l'estrema parte di un lungo processo di ristrutturazione, mirato a ripristinarne la facies paleocristiana, tanto in pianta, quanto negli alzati, processo preparato dagli scavi archeologici del 1928 e portato a compimento nel 1960, alla vigilia del Concilio Vaticano II.

La ricostruzione coinvolse l'ingresso occidentale ostruito (fig. 3), il nartece coperto da volta a botte, il livello originario del piano di calpestio, abbassato sostituendo le

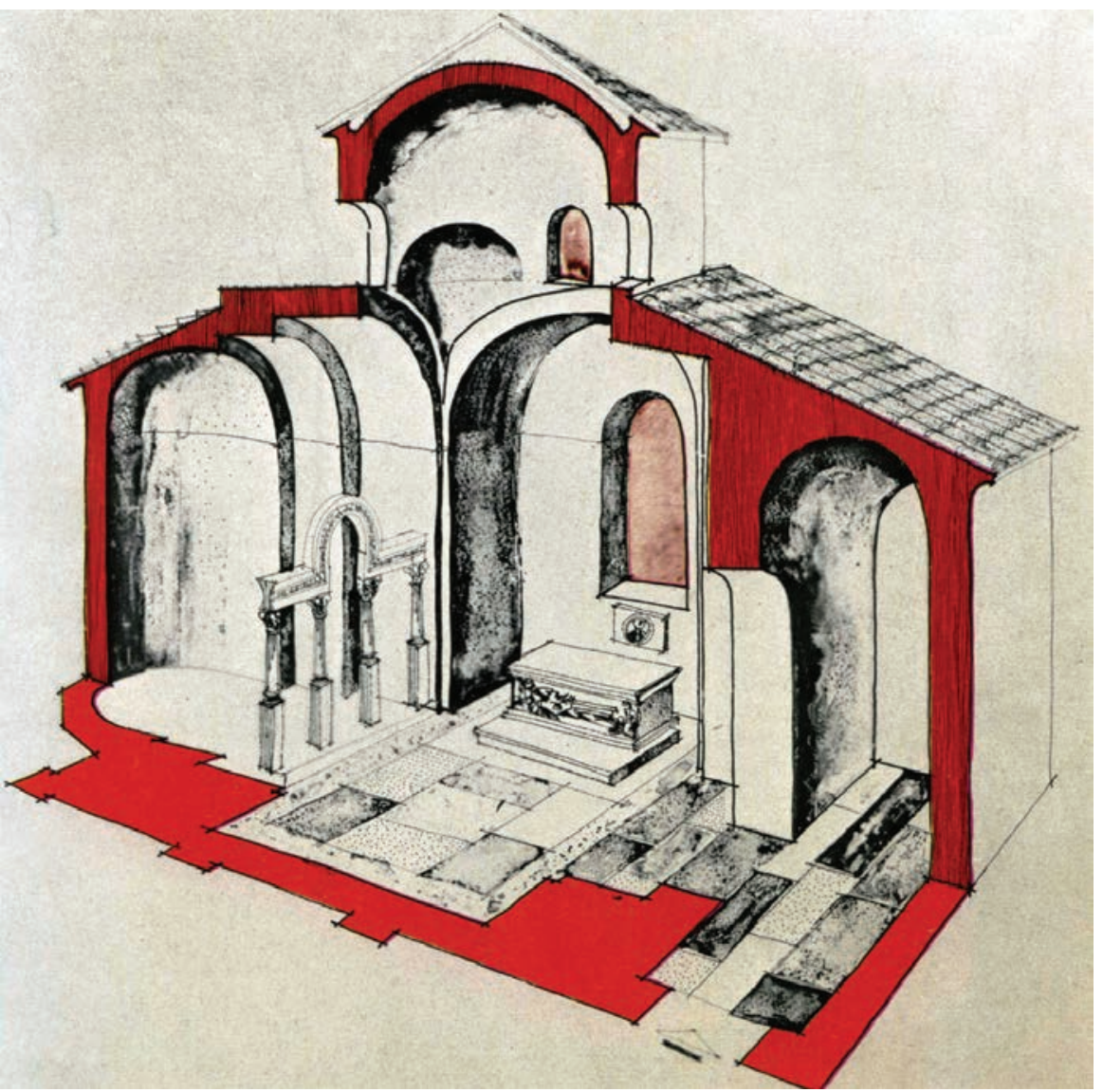

Fig. 2 Padova, Chiesa di Santa Giustina, sacello di San Prosdocimo, assonometria (da Zovatto 1970).

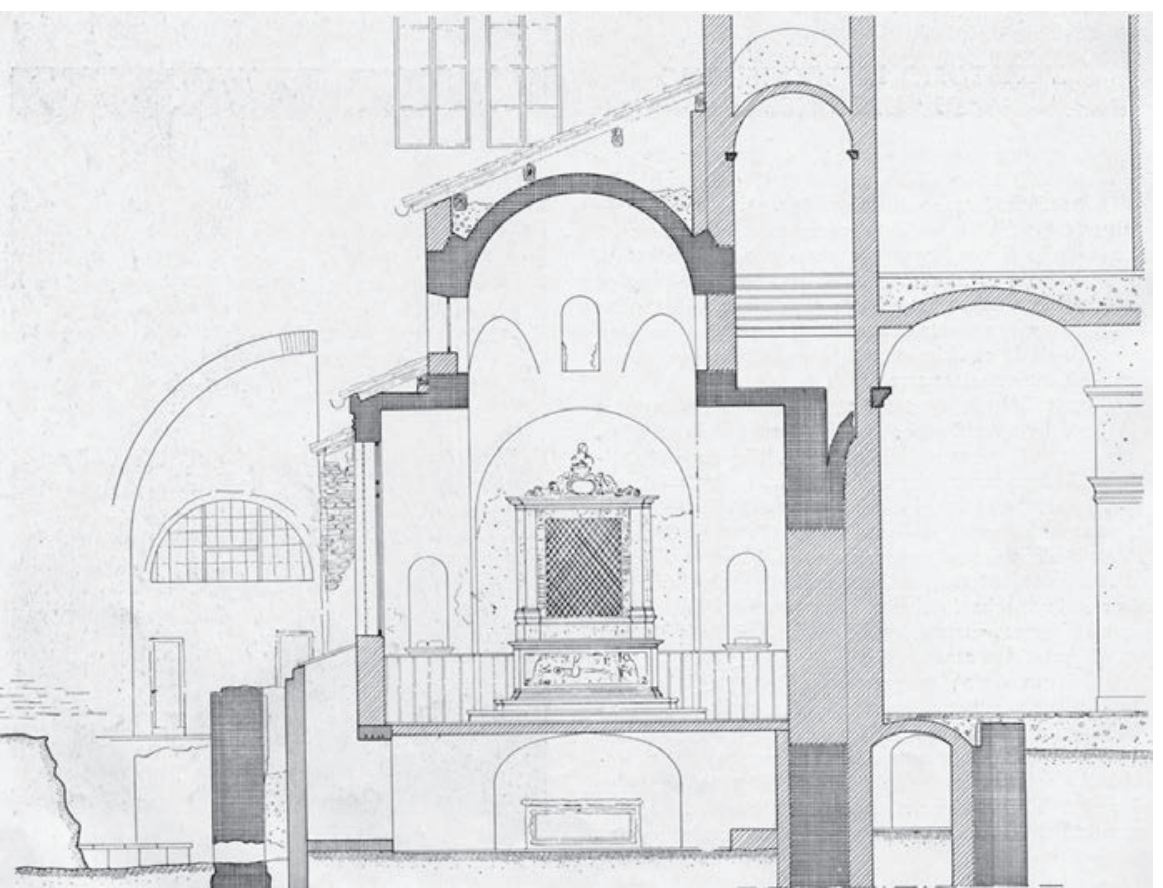

Fig. 3 Padova, Chiesa di Santa Giustina, sacello di San Prosdocimo, sezione sud (da Micheletto 1954).

volte del cimitero ipogeo con delle solide travi in cemento armato. Venne quindi posato il polito rivestimento a lastre marmoree, venne riaperta una finestra tamponata sul lato meridionale del sacello, fu ricostruita l'abside nel suo profilo

7 D. GALLO, F. ROSSETTO (ed.), Per terre e per acque: vie di comunicazione nel Veneto dal Medioevo alla prima età moderna, atti del convegno, Castello di Monselice, 16 dicembre 2001, Padova 2003; S. BORTOLAMI, Il Bacchiglione nel medioevo, in F. Selmin, C. Grandis (ed.), Il Bacchiglione, Verona 2008, pp. 141-157; E. ORLANDO, Governo delle acque e navigazione interna. Il Veneto nel basso medioevo, in Reti Medievali Rivista, 12, 2 (2011), pp. 251-93; 


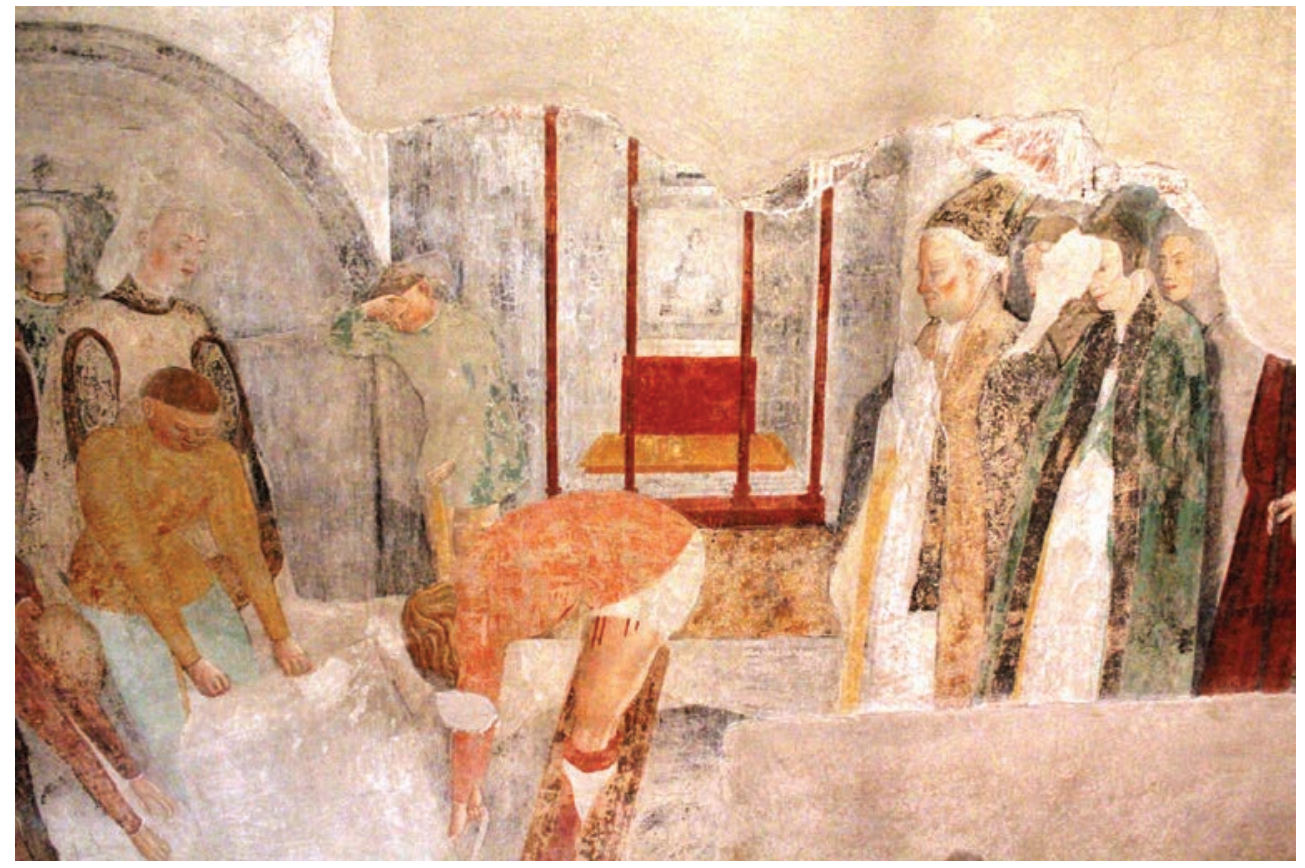

Fig. 4 Padova, Chiesa di Santa Giustina, cappella di San Luca, parete settentrionale, Giovanni Storlato, affresco, Inventio corporis di Luca evangelista nella cappella di S. Prosdocimo e di S. Maria.

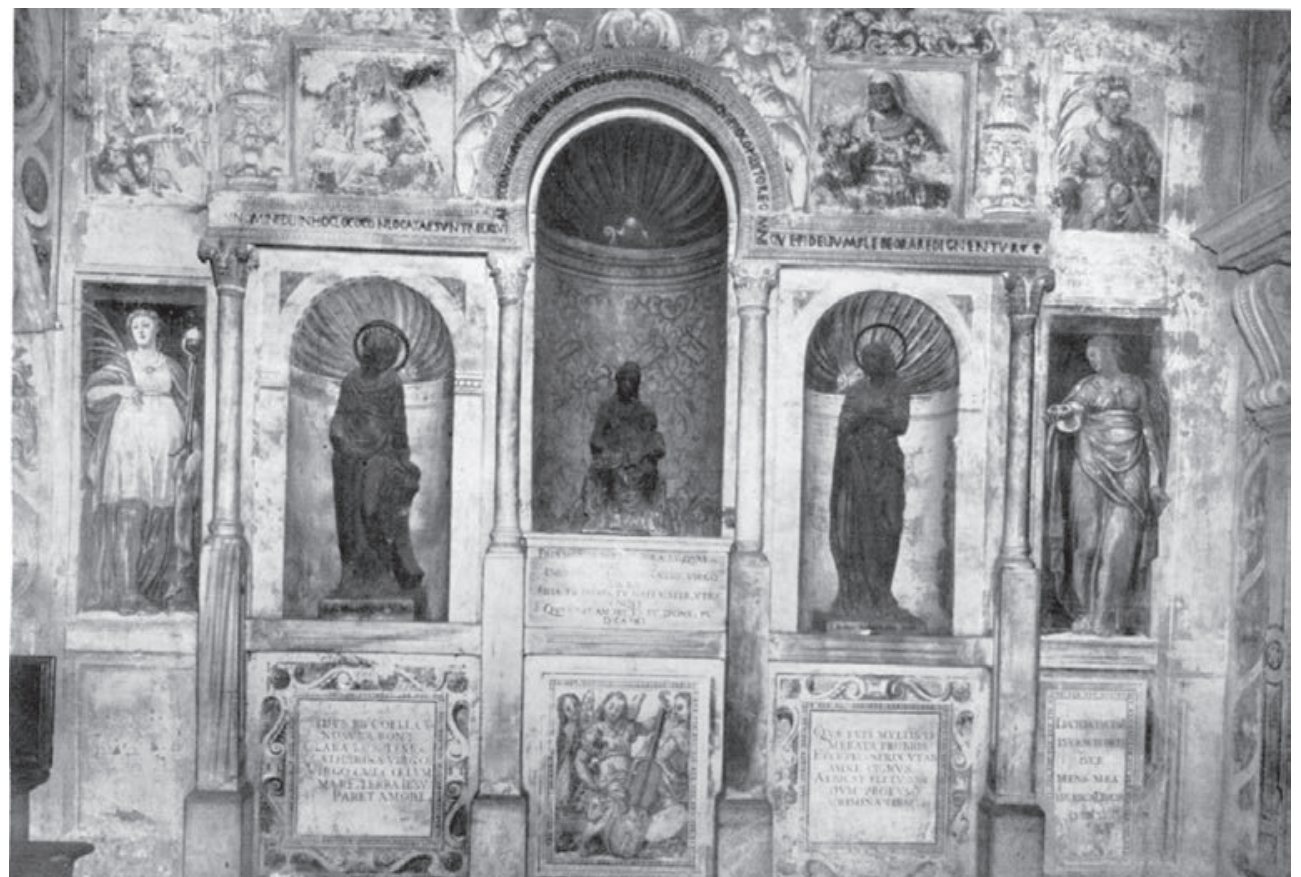

Fig. 5 Padova, Chiesa di Santa Giustina, corridoio dei martiri, foto, pergula (da Micheletto 1954).
La pergula che domina la composizione dello Storlato con il suo acceso cromatismo, venne spostata dalla galleria settentrionale del sacello, dove era stata ricoverata nel Cinquecento (fig. 5), per essere ricollocata nella sua sede originaria, a chiusura del vano absidale ricostruito nel 1961.

Si può affermare che l'unica parte del sacello che non venne interessata dalle campagne di restauro novecentesche sia la cupola (fig. 6).

Secondo l'archeologo e monsignore Paolo Lino Zovatto "gli affreschi tardo rinascimentali che hanno preso il posto dei mosaici sulle pareti del sacello, riflettono ancora i primitivi schemi decorativi e sistemi iconografici: gli apostoli nella cupola emisferica, come nel battistero degli ortodossi di Ravenna; gli evangelisti nelle trombe d'angolo, come in Santa Maria mater Domini di Vicenza e altrove"

Zovatto è testimone di una convinzione ancora viva nel 1970, ovvero quella che la cupola cinquecentesca conservasse la memoria del ciclo musivo preesistente. Nel 1932 Maria Tonzig postulava addirittura che la parte più interessante dei mosaici paleocristiani fosse rimasta in situ e semplicemente intonacata per l'esecuzione degli affreschi. Ciò può essere confermato proprio dal fatto che nella volta "sono raffigurati in affresco i dodici Apostoli quasi che il pittore del sec. XVI abbia voluto riprodurre il mosaico sottostante" ${ }^{10}$. Anche secondo Sergio Bettini il pittore cinquecentesco ha "seguito molto liberamente, s'intende - quelle ombre di decorazione musiva, che forse ancora s'intravedevano al suo tempo"".

Dopo i lavori di restauro degli anni cinquanta e sessanta del Novecento, che portarono alla scoperta di centinaia di lacerti musivi sotto la pavimentazione cinquecentesca, non si abbandona del tutto l'idea che l'affresco con gli apostoli ricalchi una traccia musiva sottostante, anzi, si cerca di stringere ancora di più il legame tra affreschi rinascimentali e mosaici paleocristiani, ribadendo le origini orientali della cupola locale ${ }^{12}$ esternamente poligonale, aperto da una finestra in posizione centrale. La presenza di questa apertura è documentata nella Cappella di San Luca in Santa Giustina, dove l'affresco di Giovanni Storlato del 1436, raffigura proprio L'inventio corporis di Luca evangelista nella cappella di S. Prosdocimo e di S. Maria ${ }^{8}$ (fig. 4).

\footnotetext{
${ }_{8}^{8}$ A. DE NICOLÒ SALMAZO, Le storie di S. Luca e di S. Mattia di Giovanni Storlato, 1. dalla leggenda alla realtà, in G.B.F. Trolese (ed.), Riforma della chiesa, cultura e spiritualità nel Quattrocento veneto, atti del convegno per il VI centenario dalla nascita di Ludovico Barbo (1382-1443), Cesena 1984, pp. 443-62; C. LIMENTANI VIRDIS, Le storie di S. Luca e di S. Mattia di Giovanni Storlato, 2. la narrazione attualizzata, Ibid., pp. 467-473.

${ }_{9}^{9}$ P.L. ZOVATTO, op. cit. (n. 2), p. 47.

${ }^{10} \mathrm{M}$. TONZIG, op. cit. (n. 2), p. 101

${ }^{\text {" }}$ S. BETTINI, op. cit. (n. 2), pp. 232-33.

${ }^{12}$ P.L. ZOVATTO, Decorazione dell'oratorio, op. cit. (n. 2), pp. 32-39.
} 


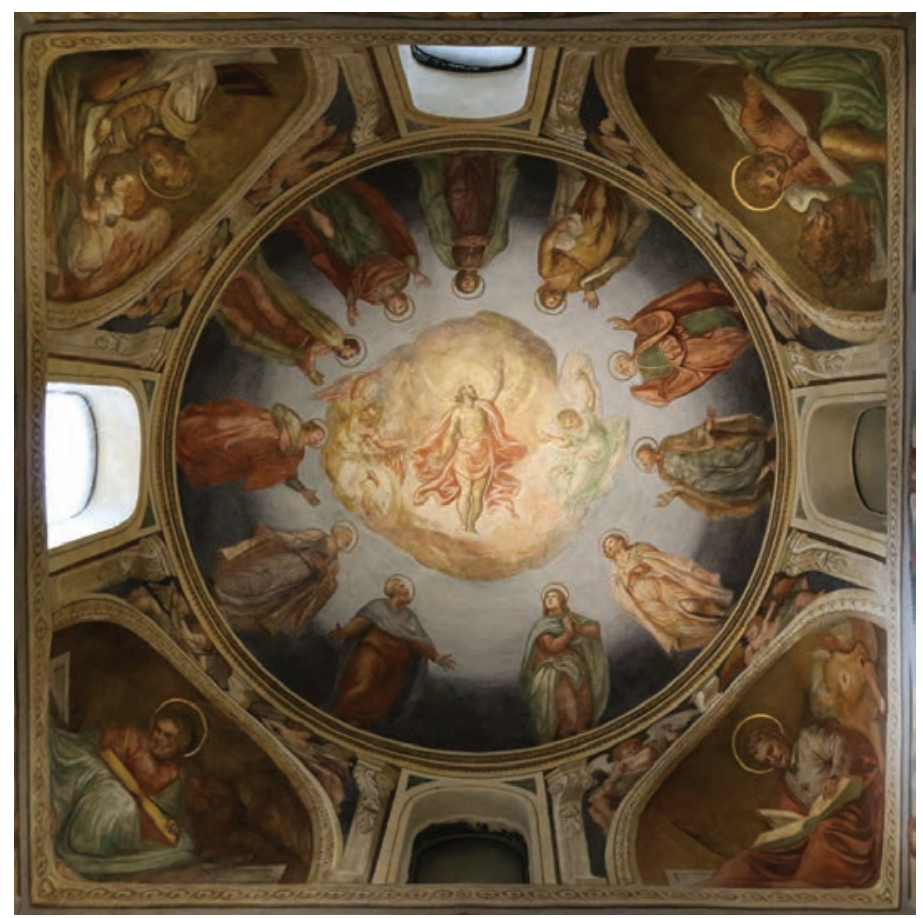

Fig. 6 Padova, Chiesa di Santa Giustina, sacello di San Prosdocimo, cupola, Camillo e Tommaso Pellegrino di Chioggia, Cornelio Campagnola, affresco, Ascensione.

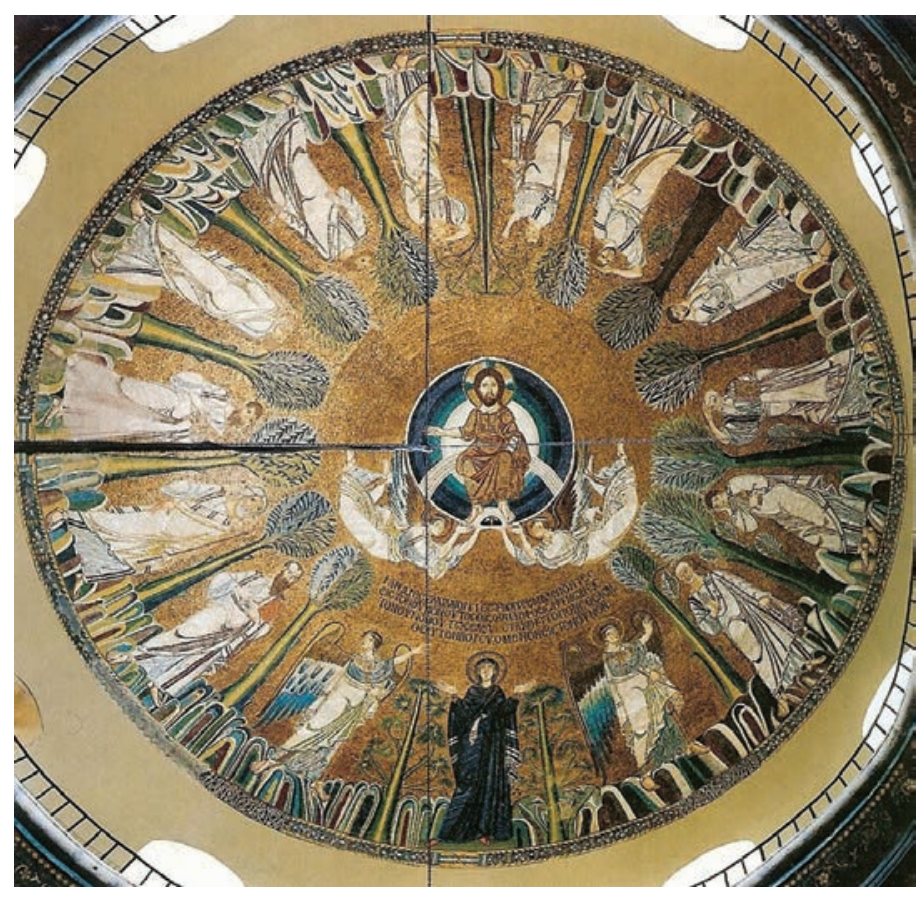

Fig. 8 Thessaloniki, Santa Sofia, cupola, mosaico, Ascensione.

che "troverebbe le sue ascendenze" in quella di San Giorgio a Salonicco, giusto perché la Leggenda di San Daniele, fonte medievale tràdita da una raccolta erudita ottocentesca sulla storia della chiesta patavina, asserisce che la volta del sacello era opere musoleo depicta quasi celeste palatium ${ }^{13}$.

Dopo un soggiorno in qualità di borsista presso l'Istituto archeologico italiano di Atene, l'architetto Leone Micheletto, laureatosi nel 1956 con una tesi sul sacello patavino, torna ripetutamente a scrivere dell'oratorio. Egli si ritiene persuaso che la cupola del sacello martiriale fosse del tutto simile a

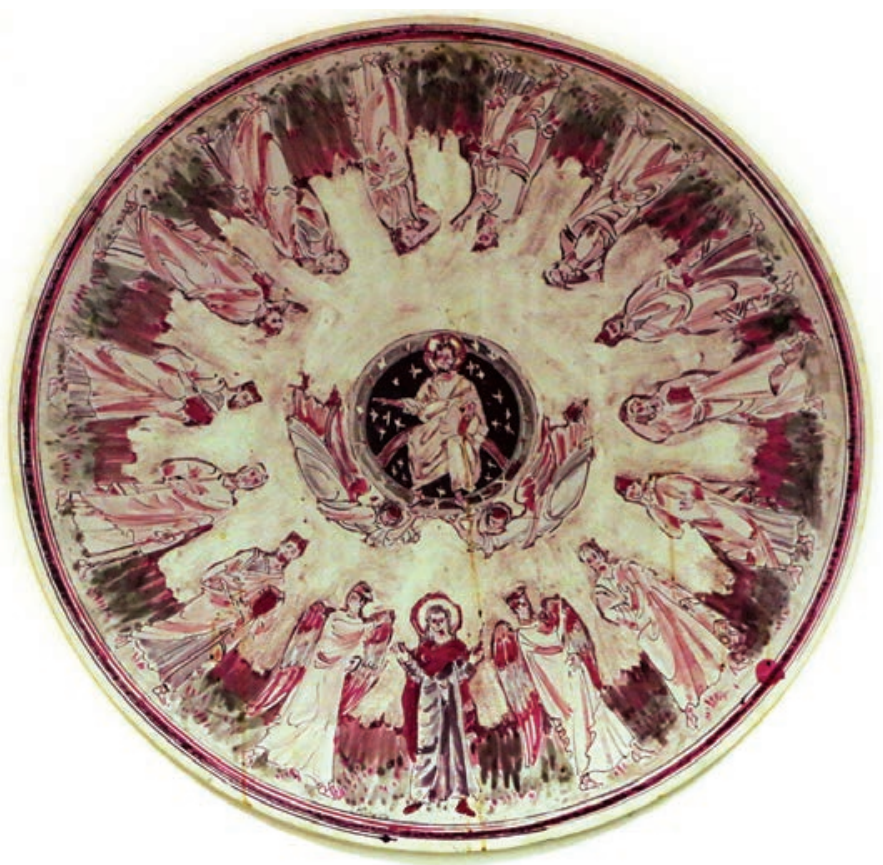

Fig. 7 Padova, Abbazia di Santa Giustina, Istituto di liturgia pastorale, Leone Micheletto, ricostruzione del perduto mosaico della cupola del sacello di San Prosdocimo, riproduzione dell'acquerello originale.

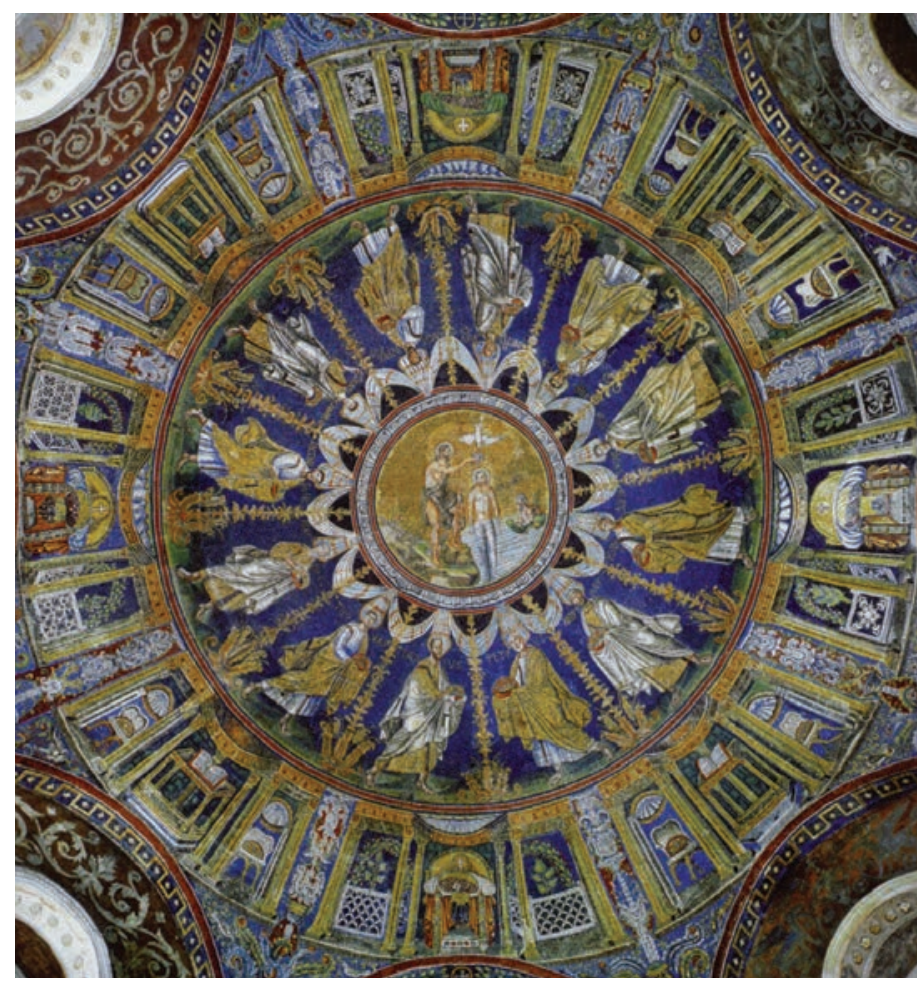

Fig. 9 Ravenna, Battistero Neoniano, cupola, mosaico, Battesimo di Cristo e Collegio apostolico.

quella della chiesa di Santa Sofia a Salonicco (fig. 8), da cui aveva tratto ispirazione per realizzare un acquerello che restituiva l'aspetto del mosaico perduto. La riproduzione di quest'opera, un tondo il cui diametro supera il metro di ampiezza, è tutt'ora conservata presso l'Istituto di liturgia pastorale di Santa Giustina ${ }^{14}$ (fig. 7).

La ricerca delle veraci antichità cristiane alle origini dell'oratorio opilioniano motivano talmente tanto gli studi

${ }^{13}$ F.S. DONDI DALL'OROLOGIO, Dissertazioni sopra i riti, discipline, costumanze della Chiesa di Padova, sino al sec. XVI, Padova 1816, diss. III, doc. XXXVI, p. 50.

${ }^{14}$ Cfr. nota 2; L. MICHELETTO, in I benedettini a Padova, op. cit. (n. 2), scheda 179, p. 306. 
novecenteschi, basati un approccio tipologico e comparativo, che, appurate le analogie fra le cupole del battistero neoniano di Ravenna (fig. 9) e di Santa Sofia a Salonicco (fig. 8), tutte caratterizzate da composizioni radiali, sfuggono dalla valutazione alcuni aspetti tutt'altro che secondari, come ad esempio il fatto che negli affreschi del sacello gli apostoli siano undici e non dodici, come vuole l'iconografia paleocristiana e bizantina, nella quale viene integrata la figura di Mattia, eletto in sostituzione di Giuda prima della Pentecoste. Inoltre non si considera che gli apostoli vestano in abiti policromi e che l'orientamento delle figure nella cupola non rispetti l'apertura originale, ma quella cinquecentesca, esito di una renovatio artistica cui venne piegato l'oratorio, nel pieno dei lavori del Concilio di Trento, al quale i monaci di Santa Giustina parteciparono attivamente ${ }^{15}$.

\section{RENOVATIO ARTISTICA CINQUECENTESCA}

Non è mai stata tentata una rilettura complessiva della renovatio artistica cui venne piegato l'oratorio nel Cinquecento, operazione che getta nuova luce sul presunto debito iconografico di Padova da Ravenna o dagli altri edifici che adottano schemi compositivi di tipo radiale sulla cupola.

Il sacello è ornato da un ciclo di affreschi commissionato nel 1564 dall'abate Angelo Faggi di Castel di Sangro (15001593) ${ }^{16}$ ai fratelli Camillo e Tommaso Pellegrino di Chioggia e a Cornelio Campagnola, secondo quanto contenuto in una nota di spesa depositata presso l'archivio di stato di Padova. Negli stessi anni, Campagnola aveva dipinto anche l'abside della chiesa del monastero benedettino di Praglia, con la stessa iconografia e con forme molto prossime a quelle adottate per la cupola patavina ${ }^{17}$.

Nella cupola del sacello di San Prosdocimo si trova l'immagine trionfale di Cristo fra undici apostoli. L'Ascensione è stata raffigurata secondo il dettato evangelico, ovvero con l'assenza di Giuda, già morto al tempo della passione e resurrezione di Gesù (fig. 6). Nelle trombe angolari sono collocati gli evangelisti insieme ai rispettivi simboli, sopra i quali, negli spazi di risulta, si trovano angeli tra le finte mensole, su cui grava la copertura. Tra queste figure e i quattro archi sottostanti si trovano motivi ornamentali a meandro, tralci abitati, interrotti da clipei che inquadrano episodi della vita di Santa Giustina e molti angeli musici che abitano le architetture dipinte.

Ma non è solo questa la parte interessata dal restauro portato a compimento nel 1564 dall'abate Angelo Faggi. Il cantiere riguarda tutto il versante meridionale della basilica di Santa Giustina, area da un lato aperta sul transetto meridionale della chiesa e dall'altra sviluppata in direzione del monastero. Egli desidera collegare alla grande fabbrica cinquecentesca gli spazi cultuali più antichi, costituiti dal sacello e dal pozzo dei martiri, che si apre sul cimitero dove furono rinvenute le sepolture di santa Giustina, martire delle persecuzioni di Massimiano, del proto-vescovo Prosdocimo, dell'apostolo Mattia e dell'evangelista Luca. Infatti il pozzo viene rivestito da marmi lavorati a niello nel 1565, il corri-

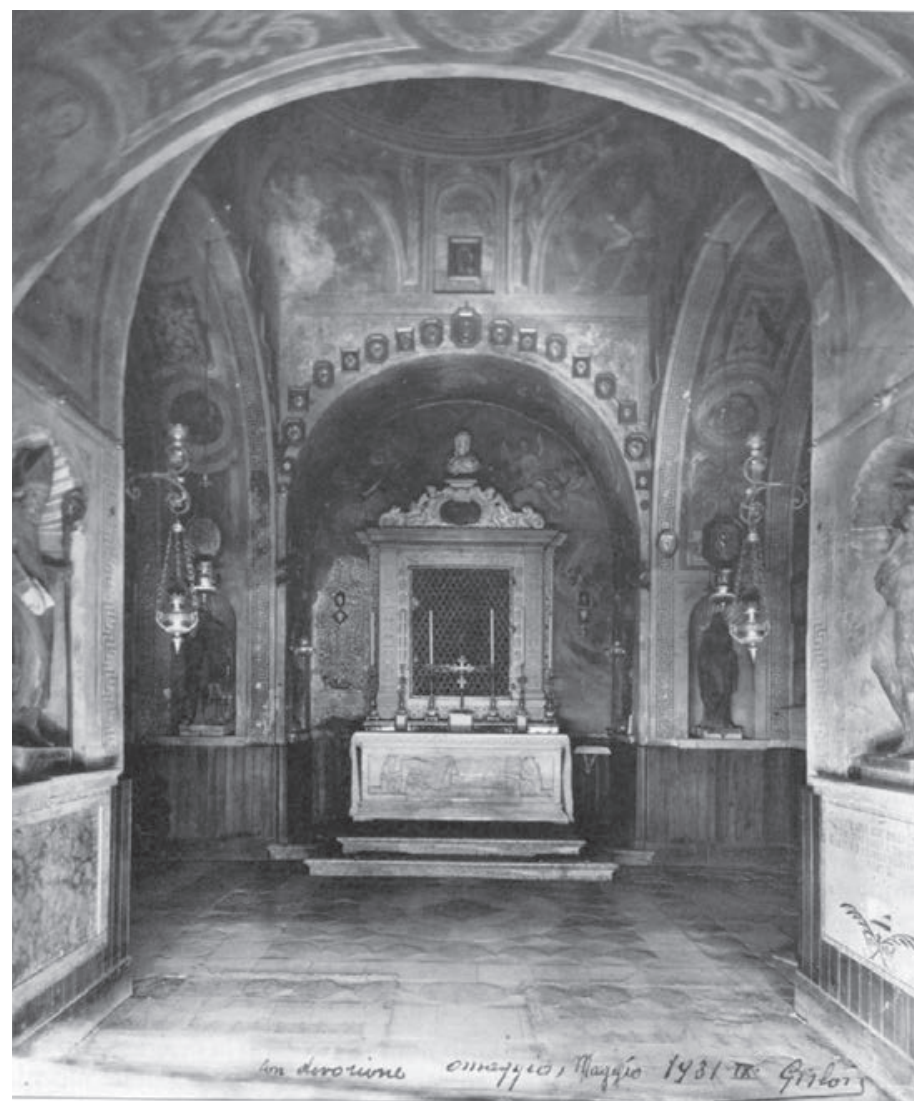

Fig. 10 Padova, Chiesa di Santa Giustina, sacello di San Prosdocimo, foto, veduta meridionale (1931).

doio viene affrescato e collegato al sacello attraverso una galleria che si interrompe all'altezza della parete orientale dell'oratorio.

In questa campagna di riallestimento il mosaico paleocristiano fu scalpellato quasi integralmente. Sono stati risparmiati solo alcuni lacerti negli intradossi delle finestre, tamponate da mattoni. Il muro orientale viene ricostruito, ma non nel rispetto delle forme precedenti. Bensì, si innalza una parete diritta che maschera l'orientamento originario dell'aula, mentre la parete occidentale viene murata, chiudendo l'originario accesso al nartece. In questo modo l'oratorio è accessibile direttamente ed esclusivamente dal lato settentrionale, dove la parete è stata sfondata, aprendo un ampio arco di passaggio fra l'oratorio e l'estremità orientale del corridoio dei martiri.

Entrando dalla nuova apertura si nota subito la fronte della cassa in cui vennero rinvenute le reliquie del proto-vescovo Prosdocimo, addossata al lato meridionale. Si tratta di un sarcofago romano di reimpiego, rilavorato dal Gallina nel 1565 con l'immagine del proto-vescovo, le cui reliquie erano state oggetto di ricognizione e traslazione ${ }^{18}$. Il valore conferito al sarcofago nell'allestimento cinquecentesco è centrale. Esso viene trattato come un paliotto d'altare, addossato al lato meridionale del sacello (fig. 10).

Modificato l'orientamento dello spazio martiriale, obliterando l'abside e spostando l'altare sul lato meridionale,

${ }^{15}$ F.G.B. TROLESE, Eutizio Cordes (1517-1585). Un monaco di Santa Giustina al concilio di Trento, in G. Spinelli (ed.), Cinquecento monastico italiano, atti del IX congresso di studi storici sull'Italia benedettina, San Benedetto Po, 18-21 settembre 2008, Cesena 2013, pp. 85-110.

${ }^{16} \mathrm{M}$. DELL'OMO, Montecassino nella chiesa e nella cultura del cinquecento, in Ibid., pp. 21-70.

${ }^{17}$ Archivio di Stato di Padova, s. Giustina, t. 491, c. 51; P.L. ZOVATTO, Decorazione dell'oratorio, op. cit. (n. 2), pp. 35-37; L. GROSSATO, Affreschi del Cinquecento in Padova, Milano 1966, pp. 185-89.

${ }^{18}$ G. ZAMPIERI, Il Sarcofago di san Prosdocimo nel contesto funerario della basilica di santa Giustina in Padova, in Un uomo, op. cit. (n. 3), pp. 45-66. 


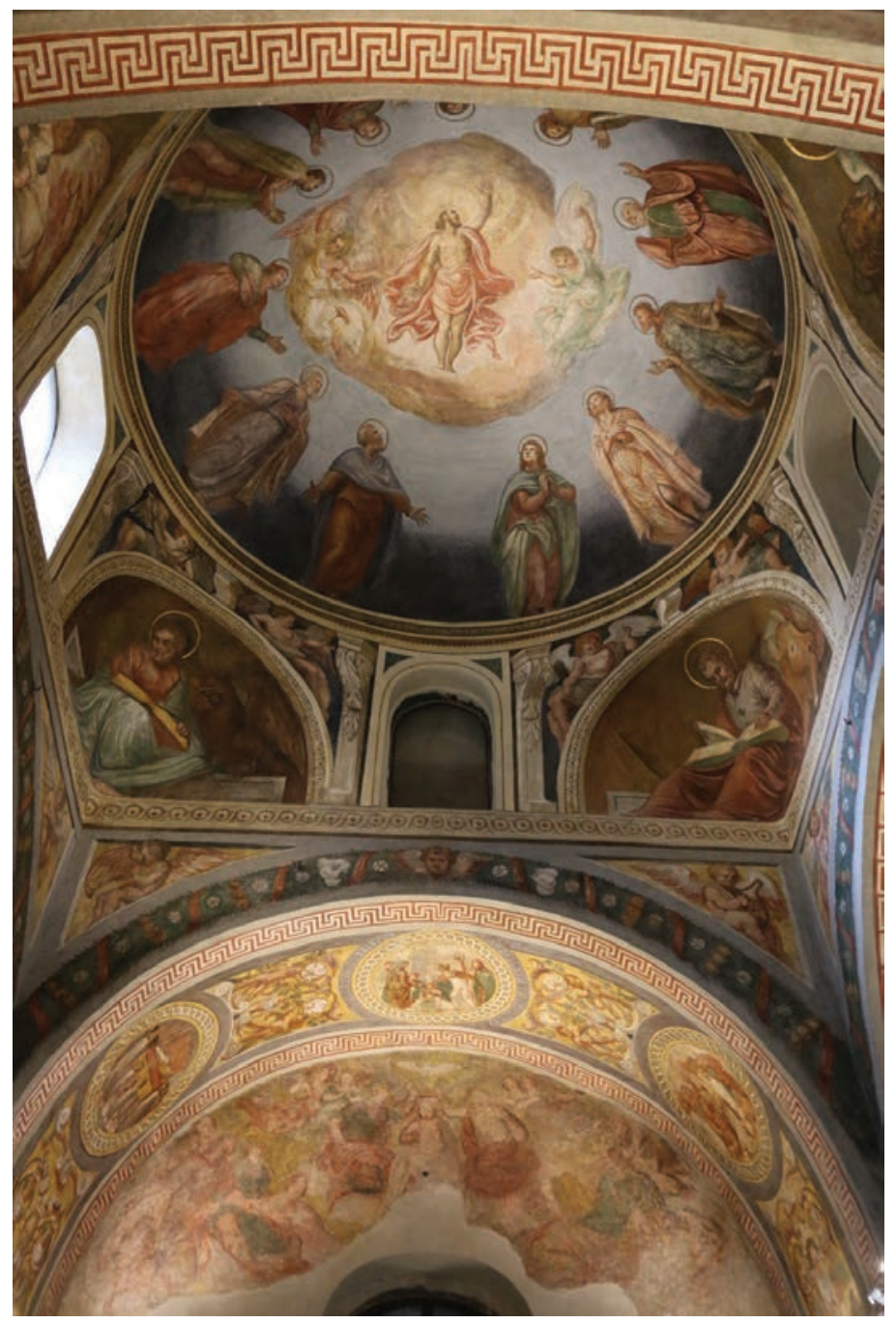

Fig. 11 Padova, Chiesa di Santa Giustina, sacello di San Prosdocimo, affreschi, veduta della lunetta meridionale e della cupola.

l'abate Angelo Faggi valorizza il culto del proto-vescovo della città, il cui sarcofago è rivolto verso la grande basilica benedettina, cui era collegato mediante il corridoio dei martiri. La programmatica celebrazione delle antichità apostoliche della città, veniva ribadita dalla pergula, collocata lungo il corridoio, di fronte al sarcofago. L'iscrizione sorretta dalle colonnine invoca l'intercessione dei santi apostoli e dei molti martiri le cui reliquie sono posate in hoc loco, affinché si degnino di pregare per il committente e per tutti i fedeli ${ }^{19}$ (fig. 5). Questo ricollocamento della pergula è del tutto congruente con il nuovo allestimento: l'altare del santo confessore, proto-vescovo della città, si trova in questo modo in posizione frontale rispetto alla pergula, che invoca gli apostoli e i martiri, ponendo Prosdocimo in asse con una tradizione apostolica che culmina nella cupola.

Proprio la cupola costituisce la chiave di volta di questo processo di risemantizzazione dello spazio martiriale. Infatti, contrariamente a quanto scrisse Leone Micheletto nel $1980^{20}$, gli affreschi dei fratelli Pellegrini di Chioggia

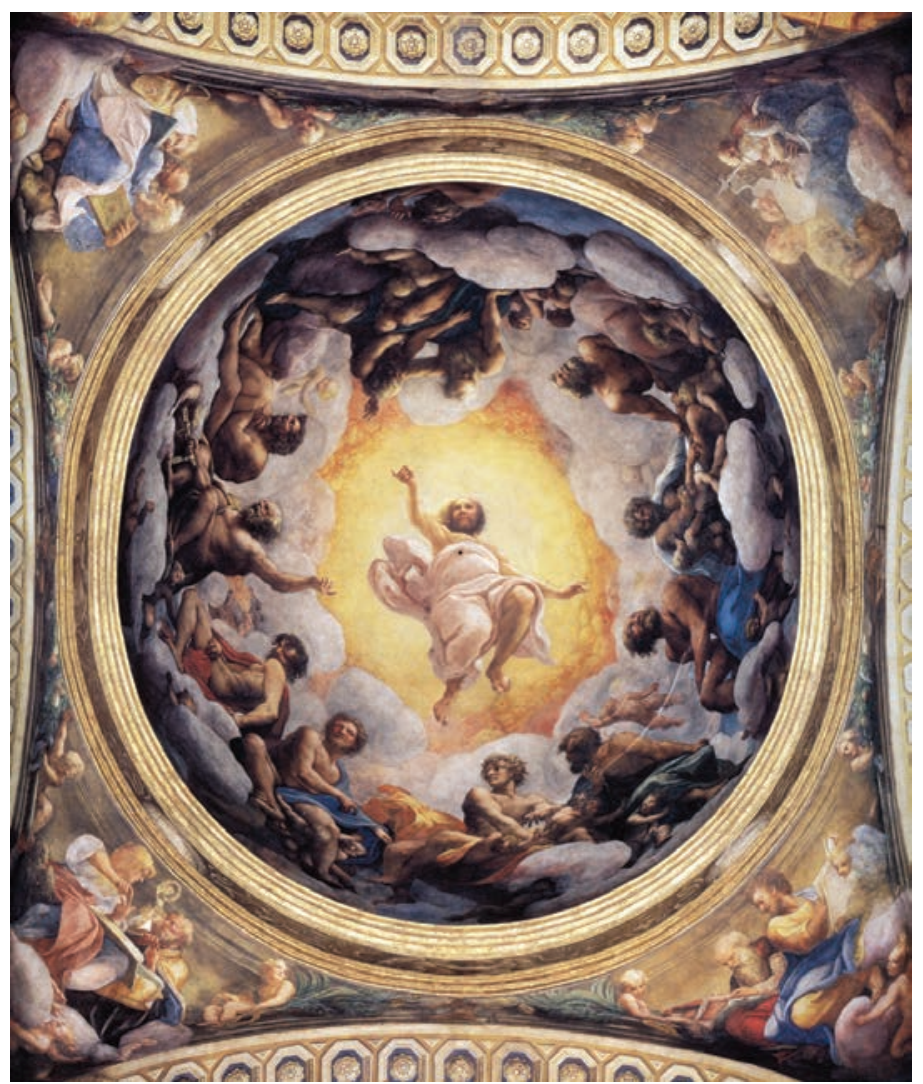

Fig. 12 Parma, Chiesa di San Giovanni, cupola, Correggio, affresco, Visione di San Giovanni.

non sono orientati lungo "l'asse paleocristiano", ovvero quello percorso da chi accedeva al sacello dall'antico nartece, muovendosi da Ovest, verso Est. Chi entri oggi nel sacello attraverso l'atrio occidentale, ricostruito nel Novecento, vede l'immagine incongrua di un Cristo "sdraiato". Per leggerlo correttamente, è necessario varcare la soglia cinquecentesca. Da questa prospettiva, la figura del Cristo trionfante domina una composizione assiale che lo pone al culmine di un climax ascendente fatto dalla figura di Giustina incoronata nella lunetta, sovrastata dal clipeo con la martire battezzata da san Prosdocimo dipinto nell'intradosso dell'arcone meridionale (fig. 11).

Gli affreschi dominano il sarcofago del protovescovo e sono rivolti verso l'accesso fatto aprire sul muro settentrionale dall'abate Sangrino. Essi quindi fanno parte integrante del processo di renovatio cinquecentesca, non sono semplicemente l'esito di un intervento mirato a ripristinare l'aspetto originario della copertura dove "erano li dodici Apostoli ma tutti affumati et annegriti dall'antiquità" ${ }^{21}$. L'abate intende valorizzare la dignità apostolica, il culto martiriale e le origini paleocristiane della basilica ${ }^{22}$, legando il sacello alla fabbrica cinquecentesca che era stata eretta più a nord, separata dall'oratorio, in origine aperto sulla chiesa paleocristiana.

L'obiettivo di rinnovare il culto locale durante gli anni del Concilio di Trento, corona un percorso avviato con Ludovico Barbo (1382-1443) più di un secolo prima. Oltre alle ragioni

\footnotetext{
${ }^{19}$ Ibid.: In nomine Dei in hoc loco conlocatae sunt reliquiae s[an]ctorum apostolorum et plurimorum martyrum qui pro conditore onmique fidelium plebe orare dignentur.

${ }^{20}$ L. MICHELETTO, in I benedettini a Padova, op. cit. (n. 2), scheda 179, p. 306.

${ }^{21}$ M. TONZIG, op. cit. (n. 2), pp. 99-100.

${ }^{22}$ B. ADORNI, L'architettura benedettina cassinese in area padana nel Rinascimento, fra koinè locale, ritorni al medioevo e disposizioni generali, in Cinquecento monastico, op. cit. (n. 15), pp. 321-40. Cfr. anche M. BONFIOLI, Tre arcate marmoree protobizantine a Lison di Portogruaro, Roma 1979.
} 
spirituali, indirizzate verso un recupero delle antichità cristiane, non sono da sottovalutare le motivazioni politiche di una tale renovatio artistica, finalizzata anche a rilanciare il ruolo della chiesa abbaziale nel territorio ${ }^{23}$.

In questo processo che coinvolge tutta l'area e il sacello con essa, l'aspetto dell'aula martiriale cambia così tanto che, secondo una cronaca del 1699, "non si conosceva più che fosse la cappella fabbricata da s. Prosdocimo"24.

Tuttavia, non si può negare il carattere arcaico degli affreschi, evidente nel contesto della pittura del secondo cinquecento in area padana. Essi paiono effettivamente riecheggiare una memoria tardoantica. L'ipotesi che costituiscano una sorta di spolia concettuale ${ }^{25}$ del perduto ciclo ornamentale è seducente e proprio per questo motivo va affrontata in modo critico. Un conto è ammettere che il ciclo ornamentale del sacello prevedesse la presenza degli apostoli, altra cosa è accettare che fossero realizzati a mosaico nella cupola come è stato ipotizzato fino ad oggi ${ }^{26}$.

\section{APOSTOLI INSCULTI?}

Il primo argomento che merita una riflessione è offerto dalle indagini archeometriche e dalla statistica: la percentuale di tessere bianche rinvenute sciolte è molto bassa, difficilmente compatibile con una teoria di apostoli nella cupola $^{27}$, posto che i confronti con le cupole a mosaico fra V e VI secolo mostrano inequivocabilmente figure vestite di tunica e pallio bianchi, oppure con drappi aurei.

Le figure in abiti policromi pertengono piuttosto alla consuetudine cinquecentesca, come si vede anche nella chiesa dell'abbazia benedettina di San Giovanni Evangelista a Parma. Nella cupola con la Visione di san Giovanni a Patmos, affrescata dal Correggio alcuni decenni prima dei rifacimenti nel sacello padovano, gli apostoli e gli evangelisti, affiancati dai dottori della Chiesa nei pennacchi, siedono coperti da stole policrome, come le tuniche. Intorno si muovono molti putti, i quali appartengono a pieno titolo alla tradizione pittorica cinquecentesca, come i cherubini della cupola padovana ${ }^{28}$ (fig. 12).

Un altro argomento che mette in discussione l'esistenza degli apostoli a mosaico in posizione radiale nella cupola viene dalla rilettura delle fonti testuali. Esse celebrano concordemente lo splendore dei mosaici aurei, ma sono tutt'altro che probanti in senso iconografico e meritano di essere riconsiderate. Infatti l'espressione depicta quasi celeste palatium costituisce un topos molto comune della letteratura cristiana, che paragona la volta degli edifici sacri ai cieli dove abita il divino ${ }^{29}$. Pertanto la fonte medievale non va presa alla lettera.

Al contrario, merita di essere adeguatamente preso in considerazione quanto affermato nella Cronica Iustiniana redatta nel $1619^{30}$. L'autore, il monaco benedettino Girolamo da Potenza, che ricevette l'abito monastico nel $1560^{31}$, fu testimone oculare dei restauri che interessarono il sacello. Nella sua descrizione della "capella antiqua di S. Prosdocimo", egli scrive che "L'Oratorio era tutto crustato da capo a basso di bellissimi lastroni. Il cielo o testudine di sopra tutto mosiato, insculti li dodici Apostoli". Questo verbo deriva dal latino insculpere, che nel Seicento viene usato con il significato di scolpire, incidere o modellare a rilievo. Il monaco di Santa Giustina non entra nel merito dell'iconografia, ma distingue chiaramente tre registri: il più basso è quello crustato, il più alto quello mosiato, quindi ci sono gli apostoli insculti.

Propongo quindi l'ipotesi che a Padova ci fossero figure in stucco come a Ravenna, nella chiesa di Santa Croce e nel battistero neoniano ${ }^{32}$.

Stando a quanto afferma Leone Micheletto, durante gli scavi realizzati a partire dagli anni quaranta del Novecento, sono stati rinvenuti molti chiodi metallici, fissati con del gesso a una distanza di trenta centimetri uno dall'altro ${ }^{33}$. Benché l'architetto considerasse l'esistenza di un sottile fregio di stucco bianco e oro, collocato tra i rivestimenti marmorei e le spalle dei finestroni, egli pensò che gli ancoraggi con tracce di gesso raccolti durante gli scavi fossero utilizzati per fissare a parete i rivestimenti lapidei ${ }^{34}$.

\footnotetext{
${ }_{23}$ F.G.B. TROLESE, Eutizio Cordes, op. cit. (n. 15), pp. 85-110: 94-95, in part. n. 48 p. 94.

${ }^{24}$ M. GERVASI, Relationi Istoriche della Chiesa e Monastero di S. Giustina di Padoa ecc., cart., 1699, ms. in Biblioteca Civica (B.P. 373, p. 72).

${ }^{25}$ M. ANDALORO, S. ROMANO, L'immagine nell'abside, in Arte e iconografia a Roma, da Costantino a Cola di Rienzo, a cura di M. Andaloro e S. Romano, Milano 2000, pp. 93-132, in part. p. 115.

${ }^{26}$ Cfr. n. 17 .

${ }^{27}$ Il dato ricavato dall'analisi della cassetta 110 ha valore statistico per due motivi. Si presume che il distacco per mezzo dello scalpello negli anni sessanta del Cinquecento sia avvenuto casualmente (le fonti dicono che gli operai agirono senza ordine). Inoltre, la testimonianza delle fonti dirette, ovvero dei monaci che nel 1957 hanno assistito di persona al ritrovamento dei lacerti musivi sotto la pavimentazione del sacello, conferma l'assenza di criteri di selezione dei materiali, raccolti con i badili, depositati nella chiesa di vicolo San Fermo a Padova e quindi ricoverati nelle 115 cassette ubicate nell'abbazia di santa Giustina. ${ }^{28}$ D. EKSERDJIAN, Correggio, Yale-Milano 1997, pp. 95-122.

${ }^{29}$ Cfr. n. 13; O. DEMUS, Byzantine mosaic decoration. Aspects of monumental art in Byzantium, London 1948; K.E. MCVEY, The domed church as microcosm: literary roots of an architectural symbol, in Dumbarton Oaks Papers, 37, 1983, pp. 91-121; S. ĆURČIĆ, Architecture as icon, in S. Ćurčić, E. Hadjitryphonos (ed.), Perception and Representation of Architecture in Byzantine Art, Exhibition at the Princeton University Art Museum, March 6, 2010-June 6, 2010, New Haven 2010, Princeton 2010, pp. 3-37; H.D. SARADI, Space in Byzantine Thought, Ibid., pp. 73-111; S. ĆURČIĆ, Divine light: constructing the immaterial in byzantine art and architecture, in B.D. Wescoat and R.G. Ousterhout (ed.), Architecture of the sacred. Space, ritual, and experience from classical Greece to Byzantium, New York 2012, pp. 307-37; Cfr. anche: M.C. CARILE, Literary Palaces. On the way to the Heavenly Jerusalem, in The vision of the Palace of the Byzantine Emperors as a Heavenly Jerusalem, Spoleto 2012, pp. 27-48.

${ }^{30}$ Cronica Iustiniana nella quale brevemente si tratta del edificazione et antiquità del Monasterio di S. Giustina e Reforma del Ordine monastico et altre cose preclare fatte in quello. Raccolto da diverse scritture antiche a comune utilità de posteri, ms., 1619, Biblioteca Civica di Padova, B.P. 829 , c. 13.

${ }^{31}$ F.G.B. TROLESE, Eutizio Cordes op. cit. (n. 15), p. 89.

${ }^{32}$ L. PASQUINI, I «gipsea metalla» di Santa Croce, in La basilica di Santa Croce. Nuovi contributi per Ravenna Tardoantica, a cura di M. David, Ravenna 2013, pp. 49-66; C. TEDESCHI, La tecnica costruttiva della cupola e i materiali utilizzati, in C Muscolino, A. Ranaldi, C. Tedeschi (ed.), Il battistero neoniano. Uno sguardo attraverso il restauro, Ravenna 2011, pp. 55-63.

33 L. MICHELETTO, L'Oratorio paleocristiano, op. cit. (n. 2), pp. 181-82.

${ }^{34}$ IDEM, in I benedettini a Padova, op. cit. (n. 2), scheda 177, p. 305.
} 
Purtroppo lo stucco è per sua natura estremamente fragile. Precipitando al suolo sotto i colpi del martello, non può che essere andato per lo più perduto, riutilizzato o ridotto a una sabbia fine contaminata con le altre malte adoperate nel sacello. Tuttavia, un'esplorazione parziale dei materiali ha portato all'individuazione di un grosso frammento di dimensioni decimetriche dal colore più bianco di quello che caratterizza le malte utilizzate per l'allettamento delle tessere. Il lacerto, inoltre, mostra una granulometria più fine e conserva ancora l'impronta di un ancoraggio metallico. Le analisi archeometriche condotte in via preliminare hanno confermato che si tratta di gesso. Questo risultato conforta l'ipotesi che tra i resti dell'oratorio paleocristiano si trovino anche frammenti di stucco.

Alla luce del ritrovamento, saranno realizzate delle prospezioni geofisiche degli alzati nel sacello. Mediante l'uso della tecnologia georadar e della termografia si potrà stimare la consistenza degli ancoraggi ancora parzialmente allettati nella malta. Mascherati dai rivestimenti marmorei e dagli affreschi, i monconi degli ancoraggi possono essere determinanti al fine di restituire l'estensione delle figure modellate in stucco e ristudiare la composizione del perduto ciclo ornamentale del sacello, con la sua articolazione in vario marmore ${ }^{35}$, apostoli in rilievo, mosaici. 